\title{
The Role of Phosphorylated Residues in Peptide-Peptide Noncovalent Complexes Formation
}

\author{
Shelley N. Jackson, Susanne C. Moyer, and Amina S. Woods \\ National Institute on Drug Abuse (NIDA) Intramural Research Program (IRP), National Institutes of Health \\ (NIH), Baltimore, Maryland, USA
}

\begin{abstract}
Electrospray mass spectrometry (ESI-MS) has become the tool of choice for the study of noncovalent complexes. Our previous work has highlighted the role of phosphorylated amino acid residues in the formation of noncovalent complexes through electrostatic interaction with arginine residues' guanidinium groups. In this study, we employ tandem mass spectrometry to investigate the gas-phase stability and dissociation pathways of these noncovalent complexes. The only difference in the three phosphopeptides tested is the nature of the phosphorylated amino acid residue. In addition the absence of acidic residues and an amidated carboxyl terminus insured that the only negative charge came from the phosphate, which allowed for the comparison of the noncovalent bond between arginine residues and each of the different phosphorylated residues. Dissociation curves were generated by plotting noncovalent complex ion intensities as a function of the nominal energy given to the noncovalent complex ion before entering the collision cell. These results showed that noncovalent complexes formed with phosphorylated tyrosine were the most stable, followed by serine and threonine, which had similar stability. (J Am Soc Mass Spectrom 2008, 19, 1535-1541) Published by Elsevier Inc. on behalf of American Society for Mass Spectrometry
\end{abstract}

$\mathrm{P}$ hosphorylation is one of the most prevalent and important post-translational modifications. Phosphorylation/dephosphorylation events function as a molecular switch for signal transduction and enzyme catalysis. In eukaryotes, serine, threonine, and tyrosine are the three amino acid residues that can be phosphorylated. Kinase consensus sites, which promote phosphorylation of amino acid residues, are some of the most abundant domains in eukaryotic organisms, thus illustrating the importance of phosphorylation [1]. Phosphorylation also greatly influences the function of proteins in which protein-phosphate interactions affect their structure [1].

The phosphate group in phosphorylated amino acid residues readily interacts noncovalently with the guanidinium group of arginine (Arg) [2]. The guanidinium group of Arg, which has a delocalized positive charge that is distributed over the entire group and a $\mathrm{p} K_{\mathrm{a}}$ of $\sim 12.5$ has a strong electrostatic attraction to the phosphate group of phosphorylated amino acid residues due to its strong negative charge and a $\mathrm{p} K_{\mathrm{a} 2}$ of $\sim 6.7$. A recent review has addressed the formation of noncovalent complexes (NCX) between the cationic guanidinium group and the anionic phosphate group [3]. The use of mass spectrometry to study noncovalent bonding between biomolecules has increased significantly. Sev-

Address reprint requests to Dr. A. S. Woods, NIDA IRP, NIH, 5500 Nathan Shock Drive, Baltimore, MD 21224, USA. E-mail: awoods@intra.nida.nih.gov eral reviews have addressed the use of electrospray mass spectrometry and matrix-assisted laser/desorption mass spectrometry to study the noncovalent interactions between protein, peptides, nucleic acids, and drugs [4-7].

In our previous work [8-11], we have demonstrated that motifs composed of at least two adjacent arginine residues on one peptide and a phosphorylated residue on the other peptide were able to generate stable NCXs that are detected by ESI-MS and MALDI-MS. In one study, it was shown that the electrostatic interaction between an Arg rich epitope and a phosphorylated one was underlying receptor heteromerization between the dopamine $\mathrm{D}_{2}$ receptor and the adenosine $A_{2 A}$ receptor [8]. In another study, the gas-phase stability of the NCXs between the basic epitope VLRRRRKRVN from the dopamine $D_{2}$ receptor and the phosphorylated/nonphosphorylated forms of an acidic epitope KVNSEEEEEDA from the NMDA receptor $\mathrm{NR}_{1}$ subunit was investigated using tandem MS [9]. The NCX with the phosphorylated form of the acidic epitope had a $60 \%$ increase in stability compared with the NCX with the nonphosphorylated form of the acidic epitope, thus demonstrating the significant increase in stability that the presence of a phosphorylated residue has on noncovalent interactions. Johnson and $\mathrm{O}^{\prime}$ Reilly have also emphasized the importance of the phosphoamino acids both in the organization of local regions of protein structure through phosphate-arginine interactions and in the 
promotion of long-range conformational responses [12]. We ascertained the effect of phosphorylation with our tandem MS studies on NCXs formed with phosphopeptides, which revealed two pathways for these complexes to separate, one in which the noncovalent interaction is dissociated and another in which the covalent bond attaching the phosphate group to the amino acid residue is fragmented, thus illustrating again the strength of electrostatic interactions involving phosphate in the gas phase $[10,11]$.

In the current study, we use ESI-MS to investigate the gas-phase stability, formation, and dissociation pathway for three phosphopeptides, (AAApSAAA, AAApTAAA, AAApYAAA) with a basic peptide (VLRRRRKRVN). Each peptide contains one of the three amino acid residues that can be phosphorylated in eukaryotes and, due to their structure the strength of the electrostatic interaction with the basic peptide, will be dependent upon the phosphorylated amino acid residue. NCXs of each phosphopeptide with the basic peptide were subjected to collision-induced dissociation at varying collision energies. Since the NCX intensities varied as a function of the nominal energy given to an NCX as it entered the collision cell, the results were used to compare the strength of the noncovalent interaction for each phosphorylated amino acid residue. Furthermore, the dissociation pathway was also compared for NCXs formed with each phosphopeptide. The results of these experiments are discussed below.

\section{Materials and Methods}

Peptides

The basic peptide VLRRRRKRVN (MW = 1351.87 Da) and three amidated phosphopeptides: AAApSAAA $(\mathrm{MW}=610.25 \mathrm{Da})$, AAApTAAA $(\mathrm{MW}=624.26 \mathrm{Da})$, AAApYAAA $(\mathrm{MW}=686.28 \mathrm{Da})$, where $\mathrm{p}$ denotes a phosphorylated residue, were synthesized at the John
Hopkins School of Medicine Peptide Synthesis Core Facility. Stock solutions were prepared in water at a concentration of $1 \mathrm{nmol} / \mu \mathrm{L}$. Sample mixtures, consisting of VLRRRRKRVN at $1 \mathrm{pmol} / \mu \mathrm{L}$ and one phosphopeptide at $7.5 \mathrm{pmol} / \mu \mathrm{L}$ or each phosphopeptide at $2.5 \mathrm{pmol} / \mu \mathrm{L}$ in $20 \%$ ethanol, were used for mass analysis.

\section{Instrument}

A Q-TOF Global Ultima mass spectrometer (Waters, Milford, MA) was used for electrospray analysis. A flow rate of $5 \mu \mathrm{L} / \mathrm{min}$ was used to introduce the sample into the mass spectrometer. The mass spectrometer was operated in positive ion mode with a capillary voltage of $3.25 \mathrm{kV}$, a sampling cone voltage of $50 \mathrm{~V}$, a source temperature of $100{ }^{\circ} \mathrm{C}$, a desolvation temperature of $300^{\circ} \mathrm{C}$, a desolvation gas flow rate of $500 \mathrm{~L} / \mathrm{h}$, and a cone gas flow of $100 \mathrm{~L} / \mathrm{h}$. For MS/MS analysis, a selection mass window of $\sim 4 \mathrm{~m} / \mathrm{z}$ with a collision gas (Argon) pressure of 13 psi was employed. Collision energies between 3 and $39 \mathrm{~V}$ were used in the collision cell for ion fragmentation. Mass spectra are the sum of 50 consecutive 1-s scans. The average intensity values reported in this study are the result of three replicate sample runs.

\section{Results and Discussion}

Initial studies were conducted to investigate the formation of NCXs of three phosphopeptides with the basic peptide VLRRRRKRVN. Figure 1 illustrates a typical ESI-MS spectrum for a sample mixture of VLRRRRKRVN and AAApYAAA, where mass peaks associated with the individual peptides were observed as follows: [VLRRRRKRVN $+3 \mathrm{H}]^{3+}$, [VLRRRRKRVN + $2 \mathrm{H}]^{2+}$, [AAApYAAA $+\mathrm{H}^{+},{ }^{+}$AAApYAAA $\left.+\mathrm{Na}\right]^{+}$, $[\text { AAApYAAA }+\mathrm{K}]^{+}$. Multi-charged peaks $\left(2^{+}, 3^{+}, 4^{+}\right)$

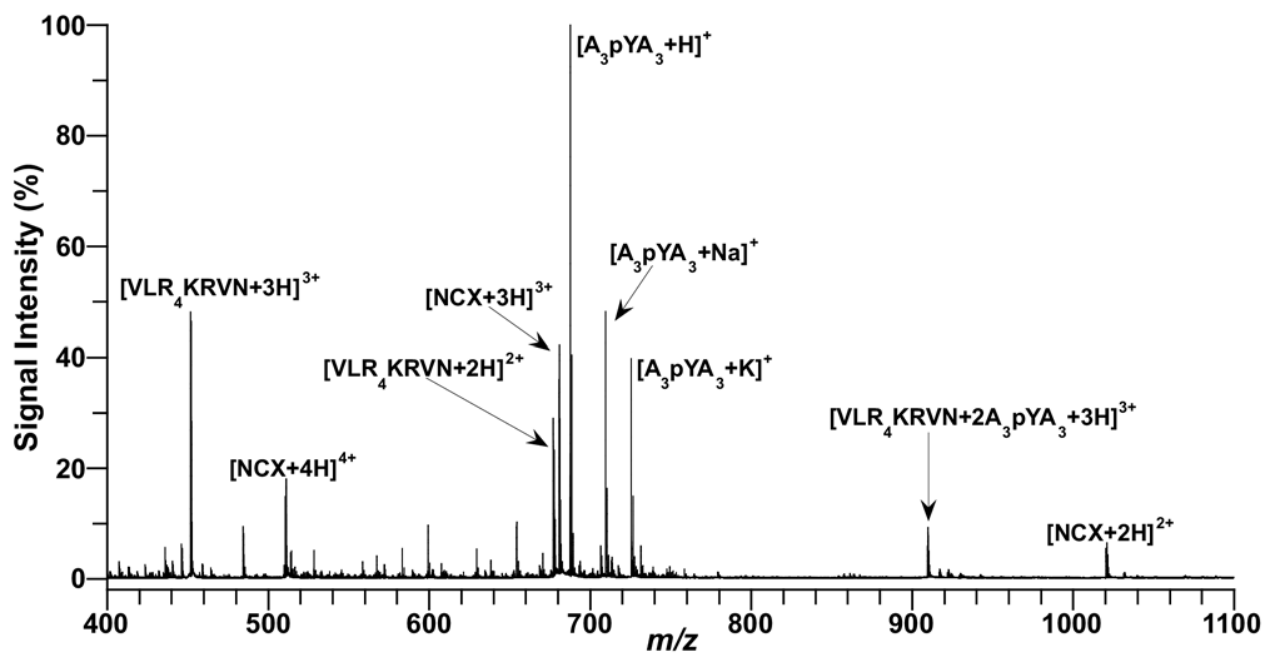

Figure 1. ESI-MS spectrum of peptide mixture consisting of $1 \mathrm{pmol} / \mu \mathrm{L}$ of VLRRRRKRVN and 7.5 $\mathrm{pmol} / \mu \mathrm{L}$ of AAApYAAA. 

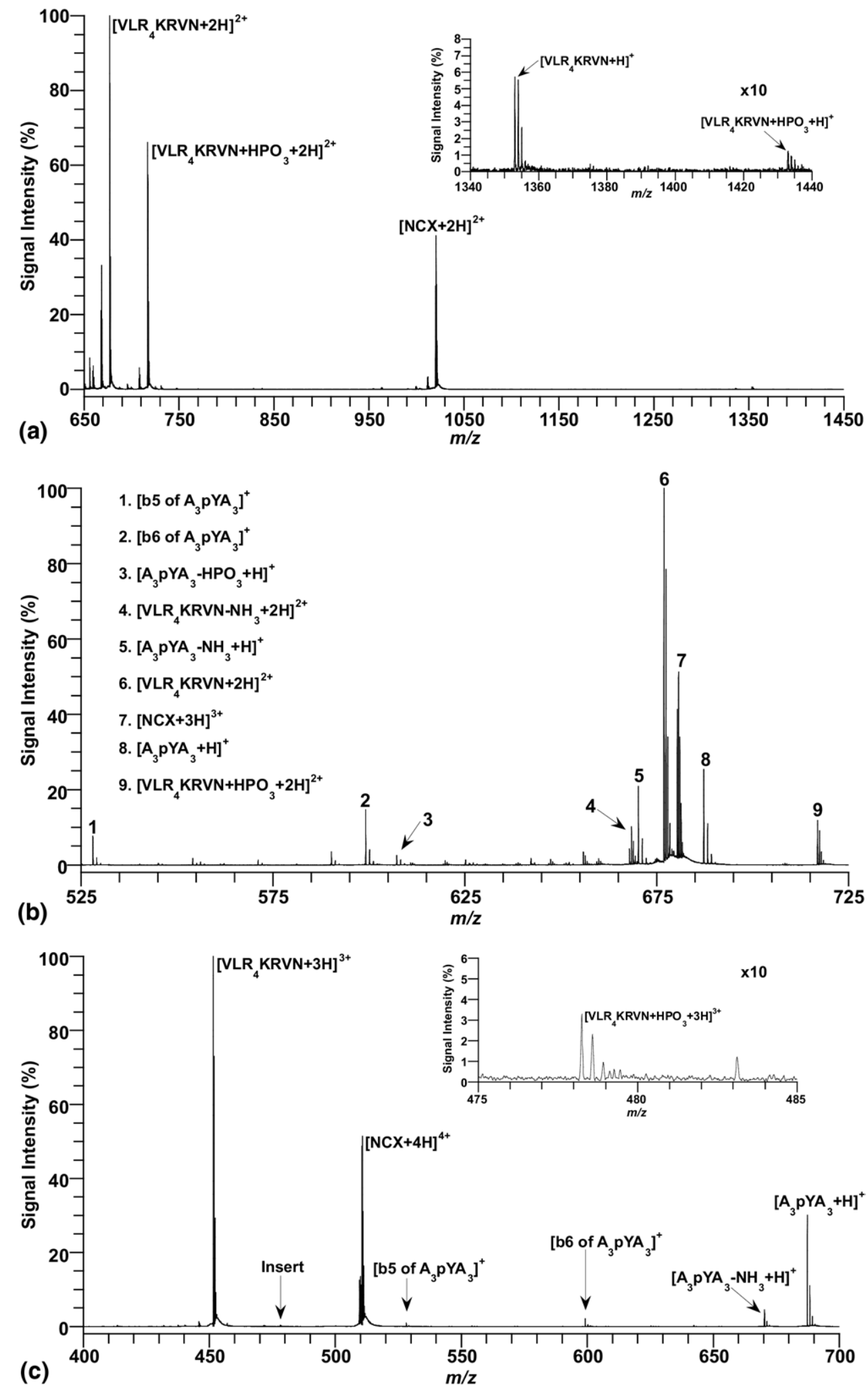

Figure 2. ESI-MS/MS spectra of NCXs of VLRRRRKRVN with AAApYAAA (a) $[\mathrm{NCX}+2 \mathrm{H}]^{2+}$ at $\mathrm{CE}=35 \mathrm{~V}$, (b) $[\mathrm{NCX}+3 \mathrm{H}]^{3+}$ at $\mathrm{CE}=20 \mathrm{~V}$, and $(\mathbf{c})[\mathrm{NCX}+4 \mathrm{H}]^{4+}$ at $\mathrm{CE}=10 \mathrm{~V}$.

were observed corresponding to the NCX formed by electrostatic interactions between the arginine residues' guanidinium group in VLRRRRKRVN and the phos- phate group of the phosphorylated tyrosine residue in AAApYAAA. Additionally, a triply charged NCX peak consisting of one VLRRRRKRVN molecule interacting 
Table 1. Ratio of VLRRRRKRVN $+\mathrm{HPO}_{3} /$ VLRRRRKRVN fragments for NCX mass peaks ${ }^{\mathrm{a}}$

\begin{tabular}{lcllrr}
\hline \multicolumn{2}{c}{$\mathrm{NCX}+2 \mathrm{H}[\mathrm{rsqb}]^{2+}$ at $25 \mathrm{~V}$} & \multicolumn{2}{c}{$\mathrm{NCX}+3 \mathrm{H}[\mathrm{rsqb}]^{3+}$ at $15 \mathrm{~V}$} & \multicolumn{2}{c}{$\mathrm{NCX}+4 \mathrm{H}[\mathrm{rsqb}]^{4+}$ at $6 \mathrm{~V}$} \\
\hline \hline AAApXAAA & Fragments $^{2+}$ & AAApXAAA & Fragments $^{3+}$ & AAApXAAA & Fragments $^{2+}$ \\
AAApSAAA & $0.53 \pm 0.032$ & AAApSAAA & $0.28 \pm 0.0029$ & AAApSAAA & $0.019 \pm 0.00064$ \\
AAApTAAA & $0.83 \pm 0.035$ & AAApTAAA & $0.31 \pm 0.0025$ & AAApTAAA & $0.0080 \pm 0.00057$ \\
AAApYAAA & $1.8 \pm 0.0042$ & AAApYAAA & $0.21 \pm 0.0038$ & AAApYAAA & $0.0044 \pm 0.00026$ \\
\hline
\end{tabular}

aThe $\mathrm{VLR}_{4} \mathrm{KRVN}+\mathrm{HPO}_{3}^{2+}$ represents the fragmentation pathway in which the covalent bond is broken between the oxygen from the amino acid residue and the phosphorus of the phosphate and the $\mathrm{VLR}_{4} \mathrm{KRVN}^{2+}$ represents the dissociation pathway in which the electrostatic interaction is disrupted. Thus, a higher value represents a stronger electrostatic interaction for the complex.

with two molecules of AAApYAAA was also detected. Similar mass spectra were observed for VLRRRRKRVN and the other two phosphopeptides (AAApSAAA and AAApTAAA).

To better understand peptide-peptide interactions involving phosphorylated amino acid residues, ESIMS/MS was conducted on the $[\mathrm{NCX}+2 \mathrm{H}]^{2+},[\mathrm{NCX}+$ $3 \mathrm{H}]^{3+}$, and $[\mathrm{NCX}+4 \mathrm{H}]^{4+}$ mass peaks formed with each phosphopeptide. In all cases, two pathways of separation were observed for the NCX. One pathway is the dissociation of the electrostatic interaction between the Arg residues and the phosphate groups, while in the alternative pathway the complex is fragmented along the covalent bond between the oxygen from Ser, Thr, or Tyr and the phosphorus of the phosphate. Similar pathways have been previously observed for NCXs formed by Arg and phosphorylated amino acid residues [10, 11].

Figure 2 shows product-ion spectra of the multicharged mass peaks $\left(2^{+}, 3^{+}, 4^{+}\right)$of the NCX between VLRRRRKRVN and AAApYAAA. Figure 2a illustrates a MS/MS spectrum at a collision energy of $35 \mathrm{~V}$ for the $[\mathrm{NCX}+2 \mathrm{H}]^{2+}$ mass peak. The two major fragment peaks observed for the NCX were [VLRRRRKRVN + $2 \mathrm{H}]^{2+}$ and [VLRRRRKRVN $\left.+\mathrm{HPO}_{3}+2 \mathrm{H}\right]^{2+}$. Minor fragment peaks were also recorded corresponding to [VLRRRRKRVN $+\mathrm{H}]^{+}$and [VLRRRRKRVN $+\mathrm{HPO}_{3}+$ $\mathrm{H}$ ] (shown in insert) and [AAApYAAA $+\mathrm{H}]^{+}$. Figure $2 \mathrm{~b}$ shows a MS/MS spectrum at a collision energy of 20 $\mathrm{V}$ for the $[\mathrm{NCX}+3 \mathrm{H}]^{3+}$ mass peak. The dissociation of $[\mathrm{NCX}+3 \mathrm{H}]^{3+}$ peak resulted in singly charged ions corresponding to AAApYAAA and doubly charged ions of VLRRRRKRVN. The fragment peaks assigned to AAApYAAA were as follows: [AAApYAAA $+\mathrm{H}^{+}$, [AAApYAAA $\left.-\mathrm{NH}_{3}+\mathrm{H}\right]^{+}$, [AAApYAAA $-\mathrm{HPO}_{3}+$ $\mathrm{H}]^{+}$, [b6 of AAApYAAA $]^{+}$, and [b5 of AAApYAAA $]^{+}$. For VLRRRRKRVN, the fragment peaks recorded were as follows: [VLRRRRKRVN $\left.+\mathrm{HPO}_{3}+2 \mathrm{H}\right]^{2+}$, [VLRRRRKRVN $+2 \mathrm{H}]^{2+}$, and [VLRRRRKRVN $\left.\mathrm{NH}_{3}+2 \mathrm{H}\right]^{2+}$. Figure 2c illustrates a product-ion spectrum of $[\mathrm{NCX}+4 \mathrm{H}]^{4+}$ at a collision energy of $9 \mathrm{~V}$. The two major fragment peaks observed for the NCX were [VLRRRRKRVN $+3 \mathrm{H}]^{3+}$ and [AAApYAAA + $\mathrm{H}]^{+}$; minor peaks assigned to [VLRRRRKRVN + $\left.\mathrm{HPO}_{3}+3 \mathrm{H}\right]^{3+}$, [AAApYAAA $\left.-\mathrm{NH}_{3}+\mathrm{H}\right]^{+}$, [b6 of AAApYAAA $]^{+}$, and [b5 of AAApYAAA $]^{+}$. Similar mass spectra were observed for NCXs with the other two phosphopeptides.
Despite observing similar patterns of dissociation for all NCX ions, the ratio of the fragmentation and dissociation pathways varied according to charge state and phosphorylated amino acid residue. Table 1 shows the ratio of the peak area for VLRRRRKRVN $+\mathrm{HPO}_{3}$ (representing the pathway in which the NCX fragments along the covalent bond) and VLRRRRKRVN (representing the pathway in which the NCX dissociates along the electrostatic interaction) of the NCX ions $\left(2^{+}\right.$, $3^{+}, 4^{+}$) with the three phosphopeptides. One clear observation from this table is a decrease in this ratio as the charge state of the NCX ion increases. One explanation for this observation is that as the protonation of the complex increases, the triple resonance stabilization [3] of the arginine residues' guanidinium group decreases and the deprotonation state of the phosphate group in the phosphorylated amino acid residue decreases, thus weakening the electrostatic bond, and requiring less energy for the dissociation of the NCX. A similar pattern of NCX dissociation due to charge state has been observed for DNA duplexes [13], PNA/DNA/ PNA triplexes [14], and complexes of DNA and pentaL-arginine [15] in negative ion mode. In these studies, an increase in the charge state of the complex weakened the noncovalent interaction holding the complex together. It was inferred from theses results that as the charge increased, Coulombic repulsions increased [1315] and the number of zwitterions decreased [14]. Another interesting result in Table 1 is the strength of the electrostatic interaction between VLRRRRKRVN and AAApYAAA in the $[\mathrm{NCX}+2 \mathrm{H}]^{2+}$ ion. This complex was the only one in Table 1 in which fragmentation along the covalent bond was greater than dissociation of the electrostatic bond. A further difference observed in the MS/MS spectra in Figure 2 is the lack of signal recorded for the phosphopeptide and its fragments for the $\mathrm{NCX} 2^{+}$. One explanation for this result is that most of the phosphopeptide is being dissociated from the complex as a neutral, in contrast with the NCX $3^{+}$and NCX $4^{+}$in which a strong series of singly charged ions associated with the phosphopeptide was recorded.

Additional experiments were conducted to evaluate the stability of NCXs formed with AAApSAAA, AAApTAAA, and AAApYAAA with the basic peptide VLRRRRKRVN. In these experiments, the NCX ions $\left(2^{+}, 3^{+}, 4^{+}\right)$were fragmented with collision energies between 3 and $39 \mathrm{~V}$. The results for each of the three 

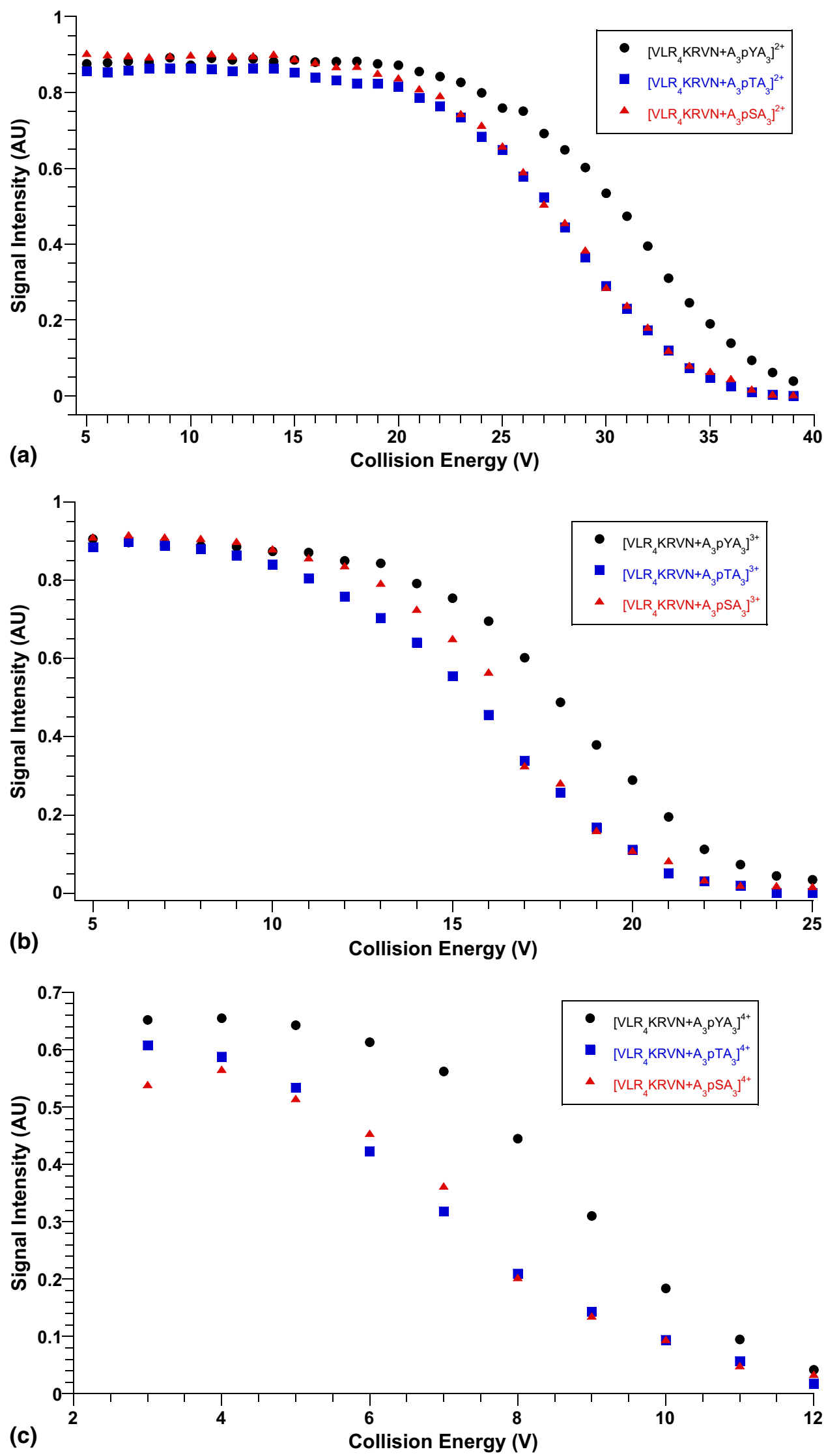

Figure 3. Stability of NCXs of VLRRRRKRVN with AAApSAAA, AAApTAAA, and AAApYAAA at varying collision energies $(a)[\mathrm{NCX}+2 \mathrm{H}]^{2+}$, (b) $[\mathrm{NCX}+3 \mathrm{H}]^{3+}$, and $(\mathbf{c})[\mathrm{NCX}+4 \mathrm{H}]^{4+}$. 
phosphopeptides are summarized in Figure 3a $[\mathrm{NCX}+$ $2 \mathrm{H}]^{2+}$, Figure $3 \mathrm{~b}[\mathrm{NCX}+3 \mathrm{H}]^{3+}$, and Figure $3 \mathrm{c}[\mathrm{NCX}+$ $4 \mathrm{H}]^{4+}$. The relative ion intensities of each complex ion in Figure 3 are plotted as a function of collision energy, using the average of three replicate measurements of ion intensity of the NCX ion. The ion intensities of each complex were normalized by dividing by the total ion count in each spectrum. For all charge states, the NCX formed with AAApYAAA was the most stable with the NCXs formed with AAApSAAA and AAApTAAA showing similar stability. One explanation for the increase stability of the NCX formed with the phosphopeptide containing tyrosine is that two different noncovalent bonds with the basic peptide are possible. One noncovalent interaction would be the electrostatic interaction between the phosphate group and a guanidinium group, and the other would be the cation- $\pi$ interaction between a guanidinium group of an arginine residue and the aromatic ring of the tyrosine side chain, with its delocalized pair of electrons. The ability of phosphorylated tyrosine residues to form noncovalent bonds with both its phosphate group and $\pi$ electrons of the benzene ring with arginine residues has been observed in X-ray crystallography data [16].

To compare affinity for each phosphorylated amino acid residue to form NCXs, ESI-MS was performed on a mixture of the three phosphopeptides and the basic peptide. The mixture consisted of VLRRRRKRVN at $1 \mathrm{pmol} / \mu \mathrm{L}$ and AAApSAAA, AAApTAAA, and AAApYAAA at $2.5 \mathrm{pmol} / \mu \mathrm{L}$ (Figure 4). For all three phosphopeptides, a strong $[\mathrm{M}+\mathrm{H}]^{+}$ mass peak and a triply-charge NCX mass peak with VLRRRRKRVN were recorded. NCX ions $\left(2^{+}, 4^{+}\right)$were also observed. To compare NCX formation for each phosphopeptide, the ratio of the NCX peak area to the $[\mathrm{M}+\mathrm{H}]^{+}$phosphopeptide peak area was calculated. These results are listed in Table 2. For every NCX ion measured, VLRRRRKRVN formed NCXs at a higher ratio with AAApYAAA than the other two phosphopetides. One explanation for this result is that due to the extra pair of electrons and the resonance of the tyrosine benzene ring, this peptide is more electronegative. Another factor is that the side-chain length of tyrosine



Figure 4. ESI-MS spectrum of peptide mixture consisting of 1 $\mathrm{pmol} / \mu \mathrm{L}$ of VLRRRRKRVN and $2.5 \mathrm{pmol} / \mu \mathrm{L}$ of each AAApSAAA, AAApTAAA, and AAApYAAA.
Table 2. Ratio of NCX mass peak/AAApXAAA ${ }^{\mathrm{a}}$

\begin{tabular}{lccc}
\hline & {$[\mathrm{NCX}+$} & $\begin{array}{c}{[\mathrm{NCX}+} \\
3 \mathrm{H}]^{3+} \\
(\%)\end{array}$ & $\begin{array}{c}{[\mathrm{NCX}+} \\
4 \mathrm{H}]^{4+}(\%)\end{array}$ \\
AAApXAAA & $2 \mathrm{H}]^{2+}(\%)$ & $24 \pm 1.3$ & $2.0 \pm 0.29$ \\
\hline \hline AAApSAAA & $3.7 \pm 0.19$ & $24 \pm 0.51$ & $1.5 \pm 0.036$ \\
AAApTAAA & $3.1 \pm 0.20$ & $21 \pm 0.51$ & $8.7 \pm 0.72$ \\
\hline AAApYAAA & $6.6 \pm 0.53$ & $35 \pm 1.6$ & 8 \\
\hline
\end{tabular}

aThe ratio of the $\mathrm{NCX}$ peak area to the $[\mathrm{M}+\mathrm{H}]^{+}$phosphopeptide peak area was used for comparison in order to normalize the data in case of difference in ionization efficiency among the three phosphopeptides.

makes its phosphate group protrude further from the peptide backbone than the phosphate group on a serine or threonine and thus would be more accessible for NCX formation with the basic peptide. Similar to the stability experiments mentioned above, AAApSAAA and AAApTAAA showed similar results for NCX formation with VLRRRRKRVN. However, the AAApSAAA ratio of NCX formation measurements were slightly higher than those of AAApTAAA for each NCX ion recorded.

\section{Conclusions}

In this work, we studied the role of the three amino acid residues that can be post-translationally phosphorylated (Ser, Thr, Tyr) in eukaryotes and their ability to form NCXs with peptides containing adjacent Arg residues. Based upon our results, phosphorylated tyrosine was shown to form the most stable NCXs and have the strongest electrostatic attraction to peptides with Arg residues. These results were attributed to the additional electron pair provided by tyrosine's benzene ring, which can lead to formation of cation- $\pi$ noncovalent complexes between the aromatic residue and Arg [2], thus increasing the number of potential noncovalent interactions between the two peptides. In addition, the position of the tyrosine phosphate group relative to the peptide backbone makes it easier to reach by phosphorylating kinases, then a phospho-serine or threonine.

\section{Acknowledgments}

The authors acknowledge support for this research by the Intramural Research Program of the National Institute on Drug Abuse, NIH. The authors thank the Office of National Drug Control Policy (ONDCP) for instrumentation funding, without which this and other projects could not have been accomplished.

\section{References}

1. Johnson, L. N.; Lewis, R. J. Structural Basis for Control by Phosphorylation. Chem. Rev. 2001, 101, 2209-2242.

2. Woods, A. S. The Mighty Arginine, the Stable Quaternary Amines, the Powerful Aromatics, and the Aggressive Phosphate: Their Role in the Noncovalent Minuet. J. Proteome Res. 2004, 3, 478-484.

3. Schug, K. A.; Lindner, W. Noncovalent Binding Between Guanidinium and Anionic Groups: Focus on Biological- and Synthetic-Based Arginine/Guanidinium Interactions with phosph[on]ate and sulf[on]ate residues. Chem. Rev. 2005, 105, 67-114.

4. Loo, J. A. Studying Noncovalent Protein Complexes by Electrospray Ionization Mass Spectrometry. Mass Spectrom. Rev. 1997, 16, 1-23.

5. Bolbach, G. Matrix-Assisted Laser Desorption/Ionization Analysis of Non-Covalent Complexes: Fundamentals and Applications. Curr. Pharm. Des. 2005, 11, 2535-2557. 
6. Hofstadler, S. A.; Griffey, R. H. Analysis of Noncovalent Complexes of DNA and RNA by Mass Spectrometry. Chem. Rev. 2001, 101, 377-390.

7. Hofstadler, S. A.; Sannes-Lowery, K. A. Applications of ESI-MS in Drug Discovery: Interrogation of Noncovalent Complexes. Nat. Rev. Drug Discov. 2006, 7, 585-595.

8. Ciruela, F.; Burgueño, J.; Casadó, V.; Canals, M.; Marcellino, D.; Goldberg, S. R.; Bader, M.; Fuxe, K.; Agnati, L. F.; Lluis, C.; Franco, R.; Ferré, S.; Woods, A. S. Combining Mass Spectrometry and Pull-Down Techniques for the Study of Receptor Heteromerization. Direct EpitopeEpitope Electrostatic Interactions Between Adenosine $\mathrm{A}_{2 \mathrm{~A}}$ and Dopamine $\mathrm{D}_{2}$ Receptors. Anal. Chem. 2004, 76, 5354-5363.

9. Jackson, S. N.; Wang, H.-Y. J.; Yergey, A.; Woods, A. S. Phosphate Stabilization of Intermolecular Interactions. J. Proteome Res. 2006, 5, 122-126.

10. Jackson, S. N.; Wang, H.-Y. J.; Woods, A. S. A Study of the Fragmentation Patterns of the Phosphate-Arginine Noncovalent Bond. J. Proteome Res. 2005, 4, 2360-2363.

11. Woods, A. S.; Ferré, S. The Amazing Stability of the Arginine-Phosphate Electrostatic Interaction. J. Proteome Res. 2005, 4, 1397-1402.
12. Johnson, L. N.; O'Reilly, M. Control by Phosphorylation. Curr. Opin. Struct. Biol. 1996, 6, 762-769.

13. Gabelica, V.; De Pauw, E. Collision-Induced Dissociation of 16-mer DNA Duplexes with Various Sequences: Evidence for Conservation of the Double Helix Conformation in the Gas Phase. Int. J. Mass Spectrom. 2002, 219, 151-159.

14. Delvolvé, A.; Tabet, J. C.; Bregant, S.; Afonso, C.; Burlina, F.; Fournier F. Charge Dependent Behavior of PNA/DNA/PNA Triplexes in the Gas Phase. J. Mass Spectrom. 2006, 41, 1498-1508.

15. Terrier, P.; Tortajada, J.; Buchmann, W. A Study of Noncovalen Complexes Involving Single-Stranded DNA and Polybasic Compounds using Nanospray Mass Spectrometry. J. Am. Soc. Mass Spectrom. 2007, 18, 346-358.

16. Gilmer, T.; Rodriguez, M.; Jordan, S.; Crosby, R.; Alligood, K.; Green, M.; Kimery, M.; Wagner, C.; Kinder, D.; Charifson, P.; Hassell, A. H. Willard, D.; Luther, M.; Rusnak, D.; Sternbach, D. D.; Mehrotra, M. Peel, M.; Shampine, L.; Davis, R.; Robbin, J.; Patel, I. R.; Kassel, D. Burkhart, W.; Moyer, M.; Bradshaw, T.; Berman, J. Peptide Inhibitors of src SH3-SH2-phosphoprotein Interactions. J. Biol. Chem. 1994, 269, 31711-31719. 